\title{
Nonresonant Effects and Hydrogen Transition Line Shape in Cosmological Recombination Problems ${ }^{1}$
}

\author{
S. G. Karshenboim ${ }^{1,2 * *}$ and V. G. Ivanov ${ }^{3 * * *}$ \\ ${ }^{1}$ Mendeleev Institute for Metrology (VNIIM), St. Petersburg, 190005 Russia \\ ${ }^{2}$ Max-Planck-Institut für Quantenoptik, Garching, 85748 Germany \\ ${ }^{3}$ Pulkovo Astronomical Observatory, Russian Academy of Sciences, \\ Pulkovskoe sh.65, St. Petersburg, 196140 Russia \\ Received November 12, 2007
}

\begin{abstract}
Data on the fluctuations in cosmic microwave background (CMB) radiation, whose accuracy is expected to increase in the immediate future, allow the cosmological recombination of atomic hydrogen and its interaction with the CMB radiation to be studied. Nonresonant effects play an important role in these recombination processes. We consider the quantum-mechanical foundations of the nonresonant processes and present our calculations for the differential two-photon decay rates of the $3 s$ and $3 d$ levels in the hydrogen atom.
\end{abstract}

PACS numbers : 95.30.Jx; 98.70.Vc

DOI: $10.1134 / \mathrm{S} 1063773708050010$

Key words: cosmology, cosmic microwave background radiation.

\section{INTRODUCTION}

Significant progress in the technology of cosmic microwave background (CMB) detectors has allowed radio-astronomers to turn to high-accuracy observations of the CMB angular fluctuations. These observations performed with ground-based instruments, during balloon flights, and from the WMAP satellite have provided unique data on the parameters of our Universe and confirmed the main predictions of the hot Universe theory. Such observations have now become the most important source of information about the key parameters of the Universe: the Hubble constant and the densities of normal baryonic matter, dark matter, and dark energy (Spergel et al. 2006). At present, the Planck Surveyor satellite is being prepared for launch and such ground-based instruments as the South Pole Telescope and the Atacama Cosmology Telescope have been put into operation. They should increase significantly the accuracy of measuring the CMB fluctuations that arise during the recombination of hydrogen in the Universe. The accuracy of these measurements will be so high that it will exceed the accuracy of the recombination cal-

\footnotetext{
* This article was submitted by the authors in English.

** E-mail: s.g.karshenboim@vniim.ru

${ }^{* * *}$ E-mail: ivanov.vg@gao.spb.ru
}

culations included in the widely known RECFAST computer program (Seager et al. 1999, 2000).

In recent years, leading theoretical cosmologists have highlighted the necessity of improving the accuracy of calculating the hydrogen recombination in the Universe in order to be able to calculate theoretically the $\mathrm{CMB}$ power spectrum with an uncertainty of the order of $0.1-1 \%$ (Seljak et al. 2003). Such an improvement in the accuracy of theoretical models requires including a considerable number of additional atomic processes that were previously just ignored under astrophysical conditions. Such works on improving the accuracy of theoretical models have been underway for several years and various atomic processes were taken into account and studied by Dubrovich and Grachev (2005), Chluba and Sunyaev (2006, 2007), Novosyadlyj (2006), Rubiño-Martín et al. (2006), Kholupenko and Ivanchik (2006), and Wong and Scott (2007).

The multi-photon transitions from upper levels of the hydrogen atom are among the most promising candidates among the atomic processes that are capable of affecting the recombination. The necessity of allowing for the two-photon processes in this problem was stressed by Dubrovich and Grachev (2005). Subsequently it was studied by Wong and Scott (2007). This problem was also intensively investigated by Chluba and Sunyaev (2007), 
who stimulated the appearance of this paper by their numerous questions. The conclusion of the abovementioned works is that the process of two-photon cascade transitions under consideration can lead to corrections of a few tenths of a percent.

A need to take into account the finite radiative width of certain atomic levels and, at the same time, to allow for the details of the spectrum, including the line shape effects, arises in a large number of problems from various fields of physics.

The goal of this paper is, in particular, a detailed quantum-mechanical analysis of the problem and an investigation of the line wings, which, as Chluba and Sunyaev (2007) showed, play a significant role in the formation of deviations from the standard picture of recombination.

\section{THE GREEN FUNCTION AS A SUM OVER STATES}

In our treatment of the two-photon decay, we use formulas of the perturbation theory and, in particular, various representations of the Green function

$$
G_{0}(E)=\frac{1}{E-H_{0}}
$$

as a sum over states. Its traditional representation as a sum over intermediate state is

$$
G_{0}(E)=\sum_{\text {all } \lambda} \frac{|\lambda\rangle\langle\lambda|}{E-E_{\lambda}}
$$

where the eigenfunctions $|\lambda\rangle$ and eigenvalues $E_{\lambda}$ are the solutions of an unperturbed problem described by the Schrödinger equation with Hamiltonian $H_{0}$. The energy $E$ in two-photon processes turns out to be the sum (or difference) of the energy of the initial state and the energy of the absorbed (emitted) photon. Sum (2) is taken over all states $\lambda$ of the discrete and continuous spectrum; the states in the discrete part of the spectrum are assumed to be stable.

Since the eigenstates of any Hermitian operator, in particular, a Hamiltonian, correspond to real eigenvalues of the operator, the energy $E_{\lambda}$ cannot have an imaginary part that corresponds to a finite state lifetime. To take into account the finite widths, it seems natural to modify this representation for the Green function by substituting the actual energies, which for the discrete spectrum include non-zero imaginary parts, instead of the unperturbed eigenvalues. The sought-for expression then takes the form ${ }^{1}$

$$
G_{\gamma}(E)=\sum_{\text {all } \lambda} \frac{|\lambda\rangle\langle\lambda|}{E-\left(E_{\lambda}-i \gamma_{\lambda} / 2\right)},
$$

where so far we make no distinction between the perturbed and unperturbed quantities for the wave function $|\lambda\rangle$ and the real part of the energy $E_{\lambda}$.

In a number of problems for the hydrogen atom, the discrete part of the spectrum may be considered as a system of narrow levels, suggesting that $\mid E_{\lambda}-$ $E_{\lambda^{\prime}} \mid \gg \gamma_{\lambda}, \gamma_{\lambda^{\prime}}$, where $\lambda$ and $\lambda^{\prime}$ are the neighboring levels that can interfere in the sum over intermediate states in the process under study.

Equation (3) may be considered as a successful interpolation of the exact Green function, since it reproduces all of the important asymptotics. If the energy $E$ is far from the bound-level energy $E_{\lambda}$ (i.e., $\mid E-$ $\left.E_{\lambda} \mid \gg \gamma_{\lambda}\right)$, then we may neglect all widths and return to Eq. (2). If, alternatively, the energy is close to a particular resonance $\lambda$ (i.e., $\left|E-E_{\lambda}\right| \leq \gamma_{\lambda}$ ), then Eq. (3) represents the Lorentz profile that phenomenologically describes a quasi-stationary state. This is obvious if we neglect all of the contributions, except the dominant resonance term

$$
G_{\lambda}(E)=\frac{|\lambda\rangle\langle\lambda|}{E-\left(E_{\lambda}-i \gamma_{\lambda} / 2\right)} .
$$

Nevertheless, Eq. (3) cannot be considered as a proper representation ab initio. Equation (2) emerged under the assumption that all bound atomic states were stable and we can describe them by a Hermitian Hamiltonian whose eigenfunctions form a complete basis. At the same time, Eq. (4) expresses the wellknown property of the Green function as a function of the energy that it has poles at arguments equal to the bound-state energies. The poles on the real axis correspond to stable states, while the state quasistationarity means that the pole is close to the axis, with the distance from it being much smaller than the distance to the nearest pole, i.e., the level width should be much smaller than the difference between the level energies.

If Eq. (3) is considered as an asymptotic expression, then the question of whether the application of such an interpolation is, in a sense, more useful than the direct use of the corresponding asymptotics remains unclear. In particular, it is unclear: (i) whether

\footnotetext{
${ }^{1}$ We use the relativistic system of units in which $\hbar=c=1$, $e^{2}=\alpha$ is the fine-structure constant, and $m$ is the electron mass. In these units, the circular frequencies of photons $(\omega)$ are equal to their energies. Below, we consider the hydrogen atom as an example for our numerical estimations. However, in discussing the various types of corrections, we explicitly write out the nuclear charge $Z$, which is equal to unity in hydrogen, and introduce the nuclear mass $M$.
} 
the interpolation can lead to meaningful corrections in regions far from and close to the resonance; and (ii) whether it can be applied in an intermediate region.

Yet another question is related to the calculation of certain integral characteristics, such as the perturbed values of the radiative widths, for which the integration is performed over all possible energies of the emitted photons.

Being phenomenological in nature, the representation (4) is rather universal in character and is widely used in various problems. At the same time, the analysis of the applicability conditions and boundaries for this representation is based on the accuracy of the fundamental theory and is more special in character. For the hydrogen atom, the exact theory is based on quantum electrodynamics, as was first suggested by Low (1952) in his classical paper.

Below, we consider several problems in which the nonresonant corrections are important using the oneand two-photon decays of the $3 s$ and $3 d$ states in a hydrogen-like atom as an example (cf. Chluba and Sunyaev 2007)

\section{THE ONE-PHOTON AND TWO-PHOTON DECAYS OF AN ATOMIC LEVEL}

The differential decay rate for the $3 l$ state with $l=0(3 s)$ or $l=2(3 d)$ can be represented as

$$
\frac{d W(3 l \rightarrow 1 s)}{d \omega}=\frac{8}{9 \pi} \omega^{3} \omega^{\prime 3} \sum_{i, k=1}^{3}\left|b_{i k}(3 l \rightarrow 1 s)\right|^{2},
$$

where

$$
\begin{gathered}
b_{i k}(3 l \rightarrow 1 s) \\
=\left\langle 1 s\left|e r_{i}\left[G\left(E_{3}-\omega\right)+G\left(E_{3}-\omega^{\prime}\right)\right] e r_{k}\right| 3 l\right\rangle
\end{gathered}
$$

is the double emission tensor, $E_{n}=-(Z \alpha)^{2} m /\left(2 n^{2}\right)$, $G(E)$ is the electron Green function, $\omega$ and $\omega^{\prime}$ are the energies of the emitted photons that satisfy the condition

$$
\omega+\omega^{\prime}=E_{3}-E_{1} \equiv \omega_{31} .
$$

To find the total decay rate for the $3 l$ state (i.e., its width), we should integrate (5) over all possible final states:

$$
\Gamma_{3 l \rightarrow 1 s}=\frac{1}{2} \int_{\omega \leq \omega_{31}} \frac{d W(3 l \rightarrow 1 s)}{d \omega} d \omega .
$$

If this integration is performed with $G(E)=$ $G_{0}(E)$, then the result is

$$
\Gamma_{3 l \rightarrow 1 s}=C_{l} \int_{0}^{1} d y \Phi(y)
$$

where we introduced the following notation for the normalized differential decay rate:

$$
\Phi(y)=\frac{\omega_{31}}{2 C_{l}} \frac{d W(\omega)}{d \omega}=y^{3}(1-y)^{3}\left|\sum_{n \geq 2}\langle 1 s\|\rho\| n p\rangle\langle n p\|\rho\| 3 l\rangle\left[\frac{1}{1-9 / n^{2}+8 y}+\frac{1}{9-9 / n^{2}-8 y}\right]\right|^{2}
$$

and $y=\omega / \omega_{31}$. Here,

$$
C_{s}=\frac{2^{18}}{3^{13}} \frac{\alpha^{2}(Z \alpha)^{6} m}{\pi}=326.731 \cdots \times Z^{6} \mathrm{~s}^{-1},
$$

$C_{d}=C_{s} / 5, \rho=Z \alpha m r$ and $\left\langle n^{\prime} l^{\prime}\|r\| n l\right\rangle$ is the reduced dipole matrix element (see, e.g., Landau and Lifshitz 1989). The behavior of the integrands in (9) is shown in Fig. 1.

Obviously, the integrals obtained diverge for both $l=0$ and 2 . Physically, this divergence reflects the fact that the decay rate in Eq. (9) should be of the order of $\alpha^{2}(Z \alpha)^{6} m$, while we know that, in fact, it is $\Gamma(3 l)=\mathcal{O}\left(\alpha(Z \alpha)^{4} m\right)$, being essentially the result of the one-photon $3 l \rightarrow 2 p$ decay.
Meanwhile, the mentioned one-photon contribution is included in the two-photon width (8). In fact, the one-photon decay is part of the two-photon decay realized as a "cascade" where we consider a threestep process: the creation of the "real" $2 p$ state (i.e., the $3 l \rightarrow 2 p$ decay), its existence during some characteristic lifetime ("propagation"), and then its decay $(2 p \rightarrow 1 s)$. The cascade picture suggests that all three described stages are independent and, hence, the lifetime of the $3 l$ state is determined by the first process, while the two remaining stages describe the fate of the decay products and their possible correlations.

Since the intermediate $2 p$ state is included in the sum over states (10), the two-photon amplitude contains the dominant decay mode and, thus, the ex- 

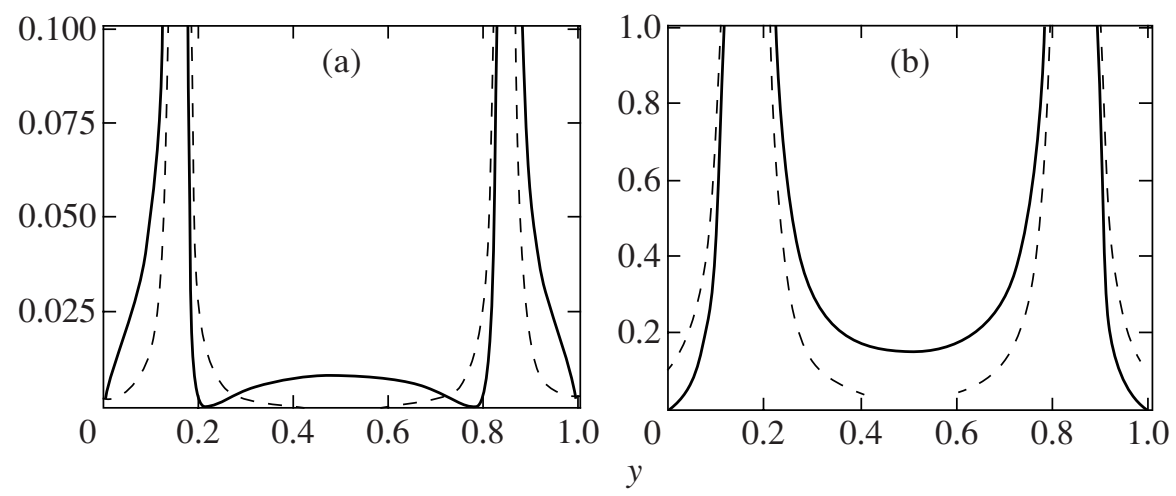

Fig. 1. Spectral profile of the normalized differential decay rate $\Phi(y)$ for the (a) $3 s$ and (b) $3 d$ states; $y=\omega / \omega_{31}$ is the relative photon energy. The resonant contour corresponding to the cascade decay is indicated by dashes.

pression for the two-photon decay should reproduce correctly the $3 l$ decay width. However, Eq. (9) cannot do this properly, because it describes incorrectly the propagation of the $2 p$ state by assuming it to be stable.

Technically, the incorrectness of describing the instability of the $2 p$ state shows up as a divergence of the term with $\lambda=2 p$ in sum (2) for $G(E)$. This contribution has poles in the integration variable $y$ at points $y_{0}=\left(E_{3}-E_{2}\right) / \omega_{31}=5 / 32$ and $y_{0}^{\prime}=1-y_{0}$. If we introduce the $2 p$ level width, then the result of the integration over frequency will become finite and we will obtain the correct (in the leading order $\left.\alpha(Z \alpha)^{4} m\right)$ result for the width.

Let us now discuss some applications of Eqs. (5) and (6) using various modifications of the representation of the Green function.

\section{CORRECTIONS TO THE TOTAL DECAY WIDTH}

If we seek for the decay width, for example, of the $3 s$ state, then the leading contribution, which is of order $\alpha(Z \alpha)^{4} m$, is determined by the one-photon $3 s \rightarrow 2 p$ E1 transition. Given the importance of the contribution with $\lambda=2 p$, it is useful to separate it out by breaking down sum (2) into three terms:

$$
\begin{gathered}
\left|\sum_{n \geq 2} \ldots\right|^{2}=|\cdots|_{n=2}^{2}+2 \operatorname{Re}\left\{[\ldots]_{n=2}\right. \\
\left.\times\left[\sum_{n>2} \ldots\right]\right\}+\left|\sum_{n>2} \ldots\right|^{2} .
\end{gathered}
$$

The first term corresponds to the "cascade" channel, the third term corresponds to the "regular twophoton" channel, and the second term corresponds to their interference. The $2 p$ level width should be introduced only in the cascade term, while the other two are regular at $\gamma_{2 p}=0$. To be more precise, it should be said that the interference term in the expression for the differential decay rate $d W / d \omega$ becomes infinite at the resonance energy for $\gamma_{2 p}=0$ and, hence, a finite $2 p$ level width is needed to make $d W / d \omega$ finite. However, the corresponding contribution to the total width $\Gamma_{3 l \rightarrow 1 s}$ is finite and we may set $\gamma_{2 p}=0$ in the integrated result (calculating the interference term near resonance is analogous to calculating a principal-value integral).

We can easily find a number of corrections to the nonrelativistic expression for the width. For instance, performing a relativistic calculation, we can obtain contributions of order $\alpha(Z \alpha)^{6} m$ (see, e.g., Sokolov and Yakovlev 1982). The recoil effects that give contributions of order $\alpha(Z \alpha)^{4}(m / M) m$ can be also taken into account (see, e.g., Fried and Martin 1963). However, all these corrections modify only the cascade contribution or, which is physically equivalent, the contribution to the one-photon decay of the $3 s$ and $3 d$ states, without affecting any two-photon effects.

The higher-order corrections are related to the two-photon contributions. For instance, using Eq. (11), we can find two-photon nonresonant corrections of order $\alpha^{2}(Z \alpha)^{6} m$, which arise from the non-cascade terms. In particular, the contribution of the "regular two-photon channel" has been calculated in various contexts previously. However, not these corrections, but the quantum-electrodynamic (QED) corrections to the resonance term (related to the corrections to both the energy and the dipole matrix element) are the most important in this order. The latter are of order $\alpha^{2}(Z \alpha)^{6} m \ln (1 /(Z \alpha))$ (Karshenboim, 1994, 1995; Ivanov and Karshenboim 1996) and generally have larger numerical coefficients than any two-photon effects. The fact that the radiative corrections to the one-photon width and the two-photons effects are formally of the same order in $\alpha$ and $Z \alpha$ is not accidental. The corrections to the 
level width in question are expressed in terms of the imaginary part of the electron two-loop self-energy in a Coulomb field, which includes both the two-photon width and the radiative QED corrections to the onephoton width. A correct value of the correction of order $\alpha^{2}(Z \alpha)^{6} \mathrm{~m}$ should be found using a complete theory (cf. Low 1952) and on no account can be obtained from an effective equations of type (3).

\section{ASYMMETRY OF THE LINE PROFILE}

Another important effect for which the corrections to the Lorentz line profile are important is the line asymmetry, which deforms the line and shifts its center (see, e.g., Fig. 1, which shows both the realistic spectrum and the resonant curves). Briefly, it can be said that the asymmetry of the line profile arises from the term of the expansion of the complete Green function near resonance following the leading one:

$$
=A\left\{\left|\frac{d W(3 l \rightarrow 1 s)(\omega)}{d \omega}\right| \frac{1}{\omega-\left(\omega_{0}-i \gamma / 2\right)}+\left.B_{1}\right|^{2}+B_{2}+\ldots\right\},
$$

where $\omega$ is the resonance energy of the $3 l \rightarrow 1 s$ decay. The coefficient $B_{1}$ is responsible for the line asymmetry and its value is determined by the total sum over states.

As applied to the $\mathrm{Ly}_{\alpha}$ line, this equation can be interpreted as follows: no resonance lines exist independently and any line is actually part of a particular cascade. Near resonance, the principal term corresponds to the Lorentz profile and its intensity is determined by the coefficient $A$, which is expressed in terms of the product of the population rate of the upper level of the transition (i.e., in our case, the $3 l \rightarrow 2 p$ decay rate) and its decay rate (in our case, the $2 p \rightarrow 1 s$ decay rate). ${ }^{2}$ Clearly, the former rate depends on the cascade and is significantly different for $3 s$ and $3 d$. In contrast, the location and width of the Lorentz profile are universal in character and only its perturbation (i.e., the coefficients $B_{1,2}$ ) depends on the cascade, which can be easily seen from Fig. 2. Usually, these corrections are referred to as the "process-dependent" corrections to the line shape.

We can consider expansion (12) by assuming that $\gamma \ll\left|\omega-\omega_{0}\right| \ll \omega_{0}$ and that the differential decay rate in this region is a smooth function of $\omega$. Because of this smoothness, we can determine the coefficients using representation (2) for the Green function and

\footnotetext{
${ }^{2}$ In a realistic case, we should consider the entire cascade, while the two-photon transition studied here is most likely the lower part of this cascade.
}

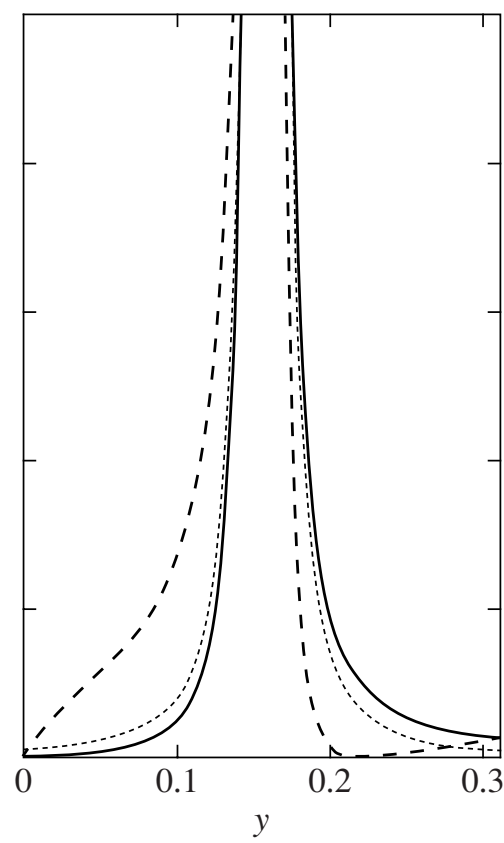

Fig. 2. Comparison of the profiles for the two-photon decay of the $3 s$ (dashes) and $3 d$ (solid line) states. The profiles were normalized to equal line intensities. The Lorentz profile is indicated by dots.

neglecting the widths of all levels (see the next section).

Thus, the overall picture corresponds to representation (3), where only the widths of the states that can be real in a given process at certain photon frequencies are assumed to be nonzero ${ }^{3}$ (only $2 p$ in the case under consideration). This approach was used, for example, by Labzowsky et al. (2001).

The far wings of the line profile, which are important for calculating the coefficients $B_{1}$ and $B_{2}$, are discussed below for the two-photon decay of the $3 \mathrm{~s}$ and $3 d$ states in the corresponding section.

\section{RELATIVISTIC EFFECTS AND LINE SHAPE}

The high-order QED effects lead to various small corrections to the parameters of Eq. (12), which are generally of little interest. The corrections to the position of the pole $\omega_{0}$, which can be important in many problems, constitute an exception. They lead to a shift of the profile as a whole without changing its shape and, in particular, the asymmetry parameter.

\footnotetext{
${ }^{3}$ At this level of accuracy, for which the high-order QED effects are disregarded, it does not matter whether the widths of the states with energies above the initial one are considered to be finite.
} 
It should be remembered that there are several degeneracies of levels with different quantum numbers in a Schrödinger hydrogen atom. The relativistic corrections lead to different shifts for different quantum numbers and, hence, remove some of the degeneracies. In particular, the $L y_{\alpha}$ line has a fine structure. There are also other, subtler effects, but the fine structure corresponds to the largest sublevel splitting $\Delta \omega_{\mathrm{FS}}$ in a nonrelativistic atom, with the distance between the fine-structure components being larger than the line width by two orders of magnitude.

The line shape (12) is valid both in the nonrelativistic approximation and in the relativistic treatment, but its parameters have different meanings. In this paper, we give explicit nonrelativistic expressions for hydrogen, which are applicable in two cases. First, they can be applied to integral characteristics, such as the total width, irrespective of whether the lines are resolvable. Second, the differential characteristics are described nonrelativistically only in the case where the detuning from resonance $\Delta \omega$ exceeds significantly the splitting and the result is averaged over spin variables. In this case, the relativistic corrections to the differential rates are of order $\left(\Delta \omega_{\mathrm{FS}} / \Delta \omega\right)^{2}$.

In particular, when the Doppler broadening is large enough, the quantities that describe real processes are "smeared" over a wide interval exceeding $\Delta \omega_{\mathrm{FS}}$, the fine-structure components are unresolvable, and their contributions are summed or averaged over spins. In this case, the nonrelativistic description is, of course, applicable.

\section{FAR WINGS OF THE LINE PROFILE}

Yet another important problem, particularly for astrophysical applications, is the description of photons far from resonance, in particular, in the region where the deviation of the photon energy from resonance (detuning), $\Delta \omega=\left|\omega-\omega_{0}\right|$, is much larger than the corresponding width $\gamma$. However, it is clear that in this region either the differential effects (at specific $\omega$ ) or some integral characteristics at which the integral over $\omega$ is taken over a region far from resonance are of interest to us.

Studying the far wings suggests that the resonance width $\gamma_{2 p}$ may be neglected and the twophoton effects were studied in this approximation in various contexts (see, e.g., Zon et al. 1968; Quatroppani et al. 1982).

Under conditions of cosmological recombination, the inhomogeneous (Doppler) broadening of the $\mathrm{Ly}_{\alpha}$ emission and absorption lines is many (approximately a hundred) times larger than the natural line width and the region in the wings of interest, as was shown by Chluba and Sunyaev (2007), extends to $10^{5} \gamma_{2 p}$.
Of course, in this case, in particular, all widths in the expression for the differential decay rate may be neglected. The Doppler broadening then turns out to be of the order of the fine structure and a more complex picture with relativistic corrections should be considered for detunings of the order of this broadening. As the detuning increases, the nonrelativistic formulas become more accurate and, in particular, at detunings of the order of a thousand $\gamma_{2 p}$, the nonrelativistic description of the differential decay rates works with an accuracy of about a percent.

Thus, for example, setting the detuning $\Delta \omega=$ $\Delta \times\left(E_{2}-E_{1}\right)$ and neglecting $\gamma_{2 p}$, we obtain the following approximation (cf. Chluba and Sunyaev 2007) for the differential decay rates moderately close to resonance $\left(\gamma_{2 p} /\left(E_{2}-E_{1}\right) \ll \Delta \ll 1\right)$ :

$$
\begin{gathered}
\frac{d W_{3 s \rightarrow 1 s}(\omega)}{d \omega} \\
=C_{s} \times\left(\frac{0.00005249}{\left(y-y_{0}\right)^{2}}-\frac{0.001601}{y-y_{0}}\right. \\
\left.+0.007482+0.02665 \cdot\left(y-y_{0}\right)+\ldots\right)
\end{gathered}
$$

and

$$
\begin{gathered}
\frac{d W_{3 d \rightarrow 1 s}(\omega)}{d \omega} \\
=C_{d} \times\left(\frac{0.002687}{\left(y-y_{0}\right)^{2}}+\frac{0.01887}{y-y_{0}}\right. \\
\left.+0.02613+0.1060 \cdot\left(y-y_{0}\right)+\ldots\right) .
\end{gathered}
$$

As we see from Fig. 3, approximations (13) and (14) work well up to a detuning of $10^{5} \gamma_{2 p}$. In terms of the representation (12), this corresponds to $B_{1}(3 s)=$ $-15.25 / \omega_{31}, \quad B_{2}(3 s)=-90.05 / \omega_{31}^{2}, \quad B_{1}(3 d)=$ $3.510 / \omega_{31}, B_{2}(3 d)=-2.598 / \omega_{31}^{2}$. The parameters should be understood as averaged over the finestructure components of the intermediate states.

The total number of photons detuned from the Lyman resonance by more than $\Delta \omega$ can be determined by the integration over the region $\omega_{0}+\Delta \times\left(E_{2}-\right.$ $\left.E_{1}\right)<\omega<\left(E_{3}-E_{1}\right) / 2$, which yields

$$
\begin{gathered}
\Gamma_{3 s \rightarrow 1 s}=C_{s}\left(\frac{0.0000622}{\Delta}+0.001601 \ln \Delta\right. \\
\left.+0.00543-0.00631 \cdot \Delta-0.00949 \cdot \Delta^{2}+\ldots\right), \\
\Gamma_{3 d \rightarrow 1 s}=C_{d}\left(\frac{0.003186}{\Delta}-0.01887 \ln \Delta\right. \\
\left.-0.00951-0.02205 \cdot \Delta-0.03774 \cdot \Delta^{2}+\ldots\right) .
\end{gathered}
$$

ASTRONOMY LETTERS Vol. $34 \quad$ No. $5 \quad 2008$ 


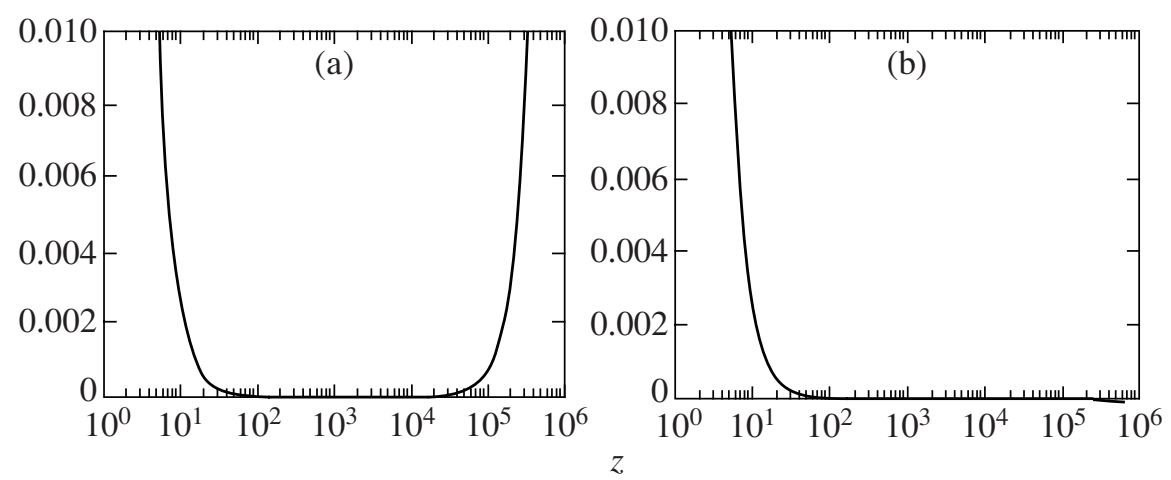

Fig. 3. Relative residual of approximations (13) (a) and (14) (b) for the spectral function $\Phi(y)$ in the wide wing of resonances. The result is the same for both peaks in Fig. 1 and, hence, $z=\left(\omega-\omega_{0}\right) / \gamma_{2 p}$ or $z=\left(\omega_{0}^{\prime}-\omega\right) / \gamma_{2 p}$.

Note that the term $\sim 1 / \Delta$ in (15) and (16) reproduces the expression

$$
\frac{1}{4 \pi \Delta \omega} \Gamma_{3 l \rightarrow 2 p} \cdot \Gamma_{2 p \rightarrow 1 s}
$$

When the frequency detuning is comparable to the line width $\gamma_{2 p} \sim \Delta \omega$, the value of (17) becomes comparable in order of magnitude to the one-photon width $\Gamma_{3 l}$. Of course, it cannot give the correct value for this width, since (16) is incorrect for $\gamma_{2 p} \sim \Delta \omega$.

\section{THE TWO-PHOTON DECAY AND CASCADE}

Above, we deliberately used the quotation marks in the words "cascade," "real" intermediate state, etc. The point is that when we have in mind the effects responsible for the one-photon decay, we should operate not with the total contribution of the $\lambda=2 p$ state, but only with its component for which the detuning frequency does not exceed several $\gamma$. In this case, the existence time of the intermediate state (i.e., the characteristic difference between the emission times of the first and second photons) is of the order of the lifetime $(\tau=1 / \gamma)$ and we actually can talk about the real $2 p$ state. This is true for the overwhelming majority of two-photon events or, in other words, for the overwhelming majority of "cascade" events with the $2 p$ state as an intermediate one.

In contrast, when we are concerned with the far wing of the Lorentz profile and consider the case where the detuning is large $(\Delta \omega \gg \gamma)$, the situation becomes qualitatively different. The characteristic time between the photon emissions turns out to be $\sim 1 / \Delta \omega$ and, hence, the $2 p$ state should more likely be understood as a virtual one.

From a physical viewpoint, there is no qualitative difference between rare events in the wings of the "cascade" contribution and the "regular two-photon" decay with an intermediate state other than $2 p$. There is no sharp boundary between the events with the real intermediate $2 p$ state (i.e., the real "cascade") and the wing events either. How we should understand the words "several $\gamma$ " is a purely practical question. To answer it and to distinguish the two situations described above, we should take into account both the necessary accuracy of the description and the various perturbations of the naive picture.

For instance, if $T$ is a certain characteristic atomic collision time, then collisions can strongly affect all of the slow two-photon events with a characteristic time $t_{\text {sep }} \geq T$. Collisions can prevent the emission of the second photon, destroy the coherence between the photons, etc. At the same time, all of the fast events with $t_{\text {sep }} \leq T$ will remain coherent and unperturbed. In particular, two coherent photons will be produced in the wings of the $2 p$ resonance for $\Delta \omega T>1$.

The natural level width is often about $10^{-6}-10^{-8}$ of the transition frequency. In such cases, the situation where the detuning frequency is much larger than the width (e.g., $10^{5} \gamma$ ), but still much smaller than the characteristic frequency can be realized. This allows all $\gamma$ in the theoretical expressions to be neglected.

It should be remembered that the natural lifetimes of all hydrogen levels (except the metastable $2 s$ level) are parametrically of the same order, namely, $\alpha(Z \alpha)^{4} m$, although numerically they can differ greatly. The upper levels generally live longer. This means that, in general, the two-photon decay includes at least three levels with different lifetimes: the initial (upper), intermediate (virtual or real) and final (lower) ones. If the lower level is neither $1 s$ nor $2 s$, then its width is generally larger than the widths of the upper and intermediate levels. If, alternatively, it is $1 s$ or $2 s$, then it is the intermediate level that is widest and we should consider it as an unstable one and, at the same time, may neglect the width of the upper state. The accuracy with which this approximation holds is determined by the smallness of the ratio of the widths. 

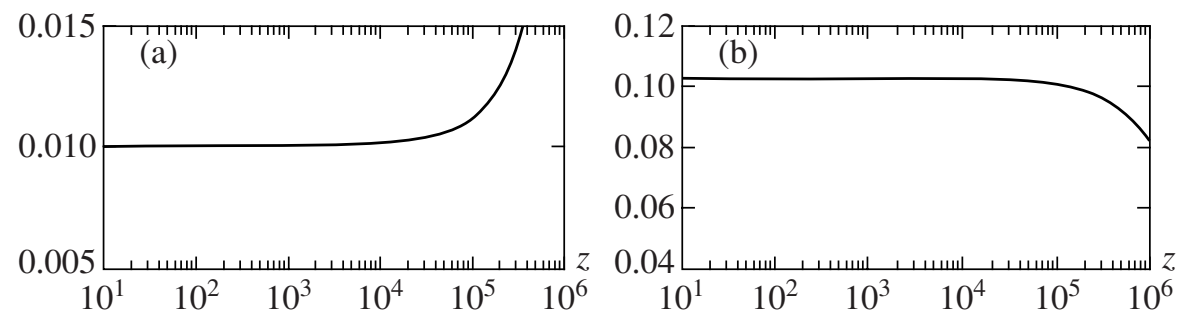

Fig. 4. Relative correction to the spectral profile in the wide wing of the Balmer resonances $3 s \rightarrow 2 p($ a) and $3 d \rightarrow 2 p$ (b) when the line width of the initial state is taken into account using (17). $z=\left(\omega-\omega_{0}\right) / \gamma_{2 p}$.

For example, for the $3 s$ and $3 d$ decays, this ratio is $\gamma_{3 s} / \gamma_{2 p} \simeq 0.01$ and $\gamma_{3 d} / \gamma_{2 p} \simeq 0.1$, respectively.

If this accuracy is not enough, then we should consider the phenomenon as a whole and study a cascade with a larger number of levels starting from and ending with states that may be considered stable.

As a rough approximation that includes the effects of a finite lifetime of the initial state, we suggest considering the energy $E_{\text {init }}$ of the $3 s$ or $3 d$ state, which is real, but is slightly shifted relative to $E_{3}$. The $E_{\text {init }}$ distribution about $E_{3}$ is described by a Lorentz profile with width $\gamma_{3 l}$,

$$
\frac{1}{2 \pi} \frac{\gamma_{3 l}}{\left(E_{\text {init }}-E_{3}\right)^{2}+\left(\gamma_{3 l} / 4\right)^{2}} .
$$

Such a treatment will not change the Lorentz profiles for the frequencies of both photons, but the sum of the frequencies will no longer be exactly equal to $\omega_{31}$, but will be only distributed about it. The wings and the nonresonant region near the Balmer peak $3 l \rightarrow 2 s$ are slightly modified (see Fig. 4), while the Lyman peak $2 p \rightarrow 1 s$ in this approximation does not change.

\section{CONCLUSIONS}

We made sure that the representation (3) for the Green function could be of use in some problems. The line asymmetry can be successfully studied using it, but it does not allow the exact total width to be found. In astrophysical applications, the bulk of the resonant profile can be successfully described by a standard Lorentz profile, while Eq. (2) is applicable far from the resonance. As a result, we conclude that the representation (3) for the Green function can be used, but it should be applied with caution. As an illustration of its application to two-photon transitions moderately close to the resonance, we presented above our results for the differential rates of the two-photon $3 s \rightarrow 1 s$ and $3 d \rightarrow 1 s$ decays in the hydrogen atom, which are of particular importance for astrophysics.

\section{ACKNOWLEDGMENTS}

This paper is largely the result of numerous discussions with J. Chluba and R.A. Sunyaev as they worked on their paper (Chluba and Sunyaev 2007). It emerged that the questions about nonresonant effects were not properly embodied in the standard literature in which there is no presentation of the formalism that would allow the astrophysical aspects of the interaction between light and hydrogen atoms to be approached from the standpoint of modern quantum mechanics. We are grateful to J. Chluba and R.A. Sunyaev for their interest in our work and their persistence. We are also grateful to L.N. Labzowsky and R.N. Lee for useful discussions. This work was supported in part by Russian Foundation for Basic Research (project nos. 06-02-16156 and 06-0204018) and DFG (grant GZ 436 RUS 113/769/0-2).

\section{REFERENCES}

1. J. Chluba and R. A. Sunyaev, Astron. Astrophys. 446, 39 (2006).

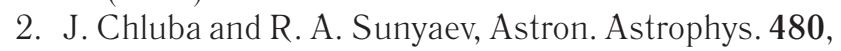
629 (2008) astro-ph/0705.3033 (2007).

3. V. K. Dubrovich and S. I. Grachev, Astron. Lett. 31, $403(2005)$.

4. Z. Fried and A. D. Martin, Nuovo Cimento 29, 574 (1963).

5. V. G. Ivanov and S. G. Karshenboim, Zh. Éksp. Teor. Fiz. 109, 1219 (1996) [JETP 82, 656 (1996)].

6. S. G. Karshenboim, Zh. Éksp. Teor. Fiz. 106, 414 (1994) [JETP 79, 230 (1994)].

7. S. G. Karshenboim, Zh. Éksp. Teor. Fiz. 107, 1061 (1995) [JETP 80, 593 (1995)].

8. E. E. Kholupenko and A. V. Ivanchik, Pis'ma Astron. Zh. 32, 883 (2006) [Astron. Lett. 32, 795 (2006)].

9. L. N. Labzowsky, D. A. Solovyev, G. Plunien, and G. Soff, Phys. Rev. Lett. 87, 143003 (2001).

10. L. D. Landau and E. M. Lifshitz, Quantum Mechanics: Non-Relativistic Theory (Nauka, Moscow, 1989; Pergamon, Oxford, 1977).

11. F. Low, Phys. Rev. 88, 53 (1952).

12. B. Novosyadlyj, Mon. Not. R. Astron. Soc. 370, 1771 (2006). 
13. J. A. Rubiño-Martín, J. Chluba, and R. A. Sunyaev, Mon. Not. R. Astron. Soc. 371, 1939 (2006).

14. S. Seager, D. D. Sasselov, and D. Scott, Astrophys. J. 523, L1 (1999).

15. S. Seager, D. D. Sasselov, and D. Scott, Astrophys. J., Suppl. Ser. 128, 407 (2000).

16. U. Seljak, N. Sugiyama, M. White, and M. Zaldarriaga, Phys. Rev. D 68, 083507 (2003).

17. Yu. L. Sokolov and V. P. Yakovlev, Zh. Éksp. Teor. Fiz. 83, 15 (1982) [Sov. Phys. JETP 56, 7 (1982)].
18. D. N. Spergel, R. Bean, O. Doré, et al., astro$\mathrm{ph} / 0603449$ (2006).

19. A. Quatroppani, F. Bassani, and S. Carillo, Phys. Rev. 25, 3079 (1982).

20. W. Y. Wong and D. Scott, Mon. Not. R. Astron. Soc. 375, 1441 (2007).

21. B. A. Zon, N. L. Manakov, and L. P. Rapoport, Zh. Éksp. Teor. Fiz. 55, 924 (1969) [Sov. Phys. JETP 28, $480(1969)]$. 